\title{
Generalized Rosenfeld scalings for tracer diffusivities in not-so-simple fluids: Mixtures and soft particles
}

\author{
William P. Krekelberg, ${ }^{1}{ }^{*}$ Mark J. Pond, ${ }^{1}$ Gaurav Goel, ${ }^{1}$ Vincent K. Shen, ${ }^{2, \dagger}$ \\ Jeffrey R. Errington, ${ }^{3, \$}$ and Thomas M. Truskett ${ }^{1,4, \S}$ \\ ${ }^{1}$ Department of Chemical Engineering, University of Texas at Austin, Austin, Texas 78712, USA \\ ${ }^{2}$ Chemical and Biochemical Reference Data Division, National Institute of Standards and Technology, Gaithersburg, \\ Maryland 20899-8320, USA \\ ${ }^{3}$ Department of Chemical and Biological Engineering, The State University of New York at Buffalo, Buffalo, New York 14260-4200, USA \\ ${ }^{4}$ Institute for Theoretical Chemistry, University of Texas at Austin, Austin, Texas 78712, USA
}

(Received 9 October 2009; published 14 December 2009)

\begin{abstract}
Rosenfeld [Phys. Rev. A 15, 2545 (1977)] originally noticed that casting the transport coefficients of simple monatomic equilibrium fluids in a specific dimensionless form makes them approximately single-valued functions of excess entropy. This observation has predictive value because, while the transport coefficients of dense fluids can be difficult to estimate from first principles, the excess entropy can often be accurately predicted from liquid-state theory. In this work, we use molecular simulations to investigate whether Rosenfeld's observation is a special case of a more general scaling law relating the tracer diffusivities of particles in mixtures to the excess entropy. Specifically, we study the tracer diffusivities, static structure, and thermodynamic properties of a variety of one- and two-component model fluid systems with either additive or nonadditive interactions of the hard-sphere or Gaussian-core form. The results of the simulations demonstrate that the effects of mixture concentration and composition, particle-size asymmetry and additivity, and strength of the interparticle interactions in these fluids are consistent with an empirical scaling law relating the excess entropy to a dimensionless (generalized Rosenfeld) form of tracer diffusivity, which we introduce here. The dimensionless form of the tracer diffusivity follows from knowledge of the intermolecular potential and the transport/thermodynamic behavior of fluids in the dilute limit. The generalized Rosenfeld scaling requires less information and provides more accurate predictions than either Enskog theory or scalings based on the pair-correlation contribution to the excess entropy. As we show, however, it also suffers from some limitations especially for systems that exhibit significant decoupling of individual component tracer diffusivities.
\end{abstract}

DOI: 10.1103/PhysRevE.80.061205

PACS number(s): 66.10.cg, 61.20.Ja, 64.70.qd

\section{INTRODUCTION}

Many computational and experimental studies have now provided empirical evidence of a strong correlation between transport coefficients and the excess entropy of equilibrium fluids (see, e.g., [1-7]). The transport coefficients (e.g., diffusivity, viscosity, and thermal conductivity) quantify the dynamic response of a fluid to a small perturbation in the associated field variables, while the excess entropy is a negative quantity that characterizes the number of microstates rendered inaccessible to the fluid (relative to an ideal gas) due to static interparticle correlations. Changes to macrostate variables that strengthen the interparticle correlations and hence make excess entropy more negative, typically result in slower dynamical processes [2]. This is true even for confined fluids $[4,8-12]$ and for systems that show anomalous dependencies of transport coefficients on density, temperature, or the strength of the interparticle attractions [13-32].

The connection between transport coefficients and excess entropy is of fundamental interest because it provides a clue

\footnotetext{
*wpkrekelberg@gmail.com

†vincent.shen@nist.gov

†jerring@buffalo.edu

${ }^{\S}$ Corresponding author; truskett@che.utexas.edu
}

in the long-standing puzzle concerning what structural and thermodynamic properties correlate with the dynamics of equilibrium fluids. The link also has practical consequences. For example, if the transport coefficients of a fluid, cast in an appropriately reduced form, can be approximately represented as a single-valued function of the excess entropy, then knowledge of the latter allows indirect "prediction" of the former [11]. The value of this approach lies in the fact that, while it is difficult to directly estimate transport coefficients from first principles, the excess entropy can often be accurately predicted from liquid-state theories.

At present, a rigorous and general statistical mechanical justification for the empirically observed relationship between transport coefficients and excess entropy is lacking. However, even in the absence of a formal justification, there are a number of practical questions that deserve further investigation. Here, we present calculations that address key aspects of two such questions.

(i) For what types of fluid systems is tracer diffusivity, when cast in an appropriately reduced form, approximately a single-valued function of excess entropy?

(ii) Can we develop a strategy for determining the aforementioned "appropriately reduced form" for tracer diffusivity of a given system from knowledge of the intermolecular potential, temperature, and composition?

To understand the context of these questions, it is helpful to first consider some background. It has long been appreci- 
ated that the following reduced form of self-diffusivity, $D^{\mathrm{R}}$ $\equiv D \rho^{1 / 3}\left(m / k_{\mathrm{B}} T\right)^{1 / 2}$, can be formally represented as a singlevalued function of excess entropy, $s^{\text {ex }}$, for any model fluid of identical particles which interact via an inverse-power-law (IPL) pair potential of the form $v(r)=\epsilon(\sigma / r)^{n}$ (see, e.g., [33]). Here, $D$ is self-diffusivity, $\rho$ is number density, $T$ is temperature, $k_{\mathrm{B}}$ is the Boltzmann constant, $m$ is the particle mass, and the combination $\epsilon \sigma^{n}$ is the single parameter of the IPL potential. The function relating $D^{\mathrm{R}}$ and $s^{\mathrm{ex}}$ strictly depends on the exponent $n$, but the dependence is weak. In fact, Rosenfeld [2] first pointed out that the relationship is "quasiuniversal" in the sense that, for a given value of excess entropy, there is less than $30 \%$ variation in the predicted self-diffusivities for different equilibrium IPL fluids with 4 $\leq n \leq \infty$. Because of this observation, we refer to $D^{\mathrm{R}}$ in this work as the Rosenfeld form of reduced diffusivity. Recently, Mittal et al. [4] demonstrated that the same quasiuniversal relationship also adequately describes the correlation between $D^{\mathrm{R}}$ and $s^{\text {ex }}$ for both bulk (isotropic) and confined (inhomogeneous) equilibrium Lennard-Jones fluids. An important point of the Rosenfeld [2] and Mittal et al. [4] investigations discussed from a different angle in a more recent study by Dyre and co-workers [34] is that since both the static and dynamic properties of many dense simple liquids are dominated by their repulsive interactions, they closely mimic the behaviors of "equivalent" IPL fluids. Thus, one can expect $D^{\mathrm{R}}$ of these monatomic simple-liquid systems to scale with $s^{\text {ex }}$ in a way that is consistent with the trend originally identified by Rosenfeld.

Yet there are many fluids that cannot be expected to mimic the behavior of monatomic IPL systems. Will excess entropy also prove useful for predicting the dynamics of these more complex fluids? For example, can excess entropy be used to reliably predict the effects of temperature and density on the self-diffusivity of model fluids with soft (or even bounded) pair potentials, such as those that characterize the effective interactions between macromolecules or micelles in solution [35-37]? At present the answer is unclear. A recent molecular-dynamics simulation study by [30] demonstrated that $D^{\mathrm{R}}$ of the Gaussian-core fluid is not (even approximately) a single-valued function of $s^{\mathrm{ex}}$, and the same is true for the Rosenfeld-scaled thermal conductivity and viscosity [38]. Is there a systematic way to construct, based on knowledge of the pair potentials and temperature of a system, an alternative reduced form, i.e., a generalized Rosenfeld diffusivity $D^{\mathrm{GR}}$, that (to within acceptable tolerances) is a function of excess entropy alone? One of the goals of this paper is to address this question for different types of model systems with a variety of interactions, [e.g., continuous or discontinuous, steeply repulsive (diverging) or soft (bounded)].

A related question is whether excess entropy can be used to predict the effects of temperature, density, and composition on the tracer diffusivities of the components of a fluid mixture? This question has been recently studied in a limited context. Specifically, following initial work on monatomic systems by [3], a scaling for the tracer diffusivities of mixtures based on two-body contributions to the excess entropy has been introduced [39]. Although it appears that this mixture version of the Dzugutov scaling can capture some of the behaviors exhibited by simple fluid systems, it also has some significant limitations. For example, the reduced diffusivity for the Dzugutov scaling, $D^{\mathrm{Z}}$, relies on defining an effective "hard-core diameter" for each interparticle potential, which is not convenient for the study of soft or penetrable particles with bounded interactions. Moreover, the Dzugutov scaling fails to describe the behavior of systems in the limit of vanishing number density. Finally, computing the two-body excess entropy requires knowledge of the radial distribution functions between all components in the mixture for each thermodynamic state of interest, which is particularly cumbersome when studying inhomogeneous fluids. This should be contrasted with the excess entropy used in the Rosenfeld scaling, which can be readily calculated from knowledge of the fluid's equation of state. For all of these reasons, we examine in this paper whether one can, based on knowledge of mixture composition, temperature, and pair potentials, construct a generalized Rosenfeld form for the reduced tracer diffusivity for component $i, D_{i}^{\mathrm{GR}}$, that is approximately a single-valued function of the excess entropy of the fluid mixture.

The organization of the paper is as follows. In Sec. II, we introduce the simple idea that underlies the generalized Rosenfeld scaling for predicting tracer diffusivity from excess entropy. Section III provides details on the model fluid systems and the simulation techniques that we use here to put the predictions of this scaling to quantitative tests. In Sec. IV, we analyze the generalized Rosenfeld scaling for a wide variety of binary mixtures of hard spheres, WidomRowlinson particles, and Gaussian-core particles. In Sec. V, we discuss how this data helps to understand the strengths and limitations of using excess entropy for predicting the effects that macroscopic parameters (temperature, density, and composition) and microscopic details (particle diameter, particle mass, and softness of the interparticle potential) have on single-particle dynamics.

\section{GENERALIZED ROSENFELD FORM FOR REDUCED TRACER DIFFUSIVITY}

In order to ensure that $D_{i}^{\mathrm{GR}}$, the generalized Rosenfeld form of the reduced tracer diffusivity of component $i$, is defined in a way that is useful for making excess-entropy based predictions, we aim to have it satisfy the following three criteria for a given system. (i) It should be proportional to the bare tracer diffusivity, i.e., $D_{i}^{\mathrm{GR}}=\alpha D_{i}$. (ii) The prefactor $\alpha$, which has units of reciprocal diffusivity, should be readily calculable based on knowledge of the parameters that define the fluid system, i.e., macroscopic variables such as temperature $T$, density $\rho$, and the mole fractions of the species, as well as microscopic parameters such as particle masses and the pair potentials $V_{i j}(r)$ describing the effective interactions. (iii) The dimensionless quantity $D_{i}^{\mathrm{GR}}$ should be approximately a single-valued function of $s^{\mathrm{ex}}$.

For the case of a monatomic IPL fluid, the aforementioned criteria are rigorously satisfied at all state points if one adopts the Rosenfeld form of the reduced self-diffusivity $D^{\mathrm{R}}$ discussed in the introduction. In fact, Rosenfeld [2] previously illustrated that, at low number density, an analytical 
relationship between $D^{\mathrm{R}}$ and $s^{\text {ex }}$ can be obtained for an IPL fluid by using two equations: an Enskog theory expression for $D$ and a truncated second-virial expansion for $s^{\text {ex }}$.

Our approach here is to similarly examine the low-density limit of more complex model fluids and mixtures, taking advantage of known theoretical results for the tracer diffusivity and excess entropy to seek out a potentially useful definition for $D_{i}^{\mathrm{GR}}$. As we show below, these low-density theoretical results do suggest a simple expression for $D_{i}^{\mathrm{GR}}$ that, in the dilute limit, satisfies the three criteria mentioned above. Of course, unlike for an IPL fluid, choosing $D_{i}^{\mathrm{GR}}$ of a complex fluid so that it is a single-valued function of $s^{\mathrm{ex}}$ at low density does not guarantee that it will also behave that way at high particle density. In fact, one can view the extent to which the $D_{i}^{\mathrm{GR}}$ versus $s^{\mathrm{ex}}$ scaling holds at higher particle density as a measure of the utility of excess entropy for predicting tracer diffusivity of a given fluid system. Section IV focuses on quantitatively examining this point for a variety of model fluids.

To make the above discussion more concrete, first consider that kinetic theory indicates that $D_{i}$ is inversely proportional to number density $\rho$ in a fluid mixture at low density [40]

$$
D_{i}=\frac{\left[\rho D_{i}\right]_{0}}{\rho},
$$

where the quantity $\left[\rho D_{i}\right]_{0} \equiv \lim _{\rho \rightarrow 0} \rho D_{i}$ generally depends on temperature, mixture composition, as well as the masses of the species and their the interparticle interactions. We discuss simple theoretical methods for estimating $\left[\rho D_{i}\right]_{0}$ for model systems below. The relationship between $\rho$ and $s^{\mathrm{ex}}$, to leading order in $\rho$, can be expressed as

$$
s^{\mathrm{ex}} / k_{\mathrm{B}}=-\rho\left[B+T \frac{d B}{d T}\right],
$$

where $B$ is the second virial coefficient given by

$$
B=\sum_{i}^{N_{c}} \sum_{j}^{N_{c}} x_{i} x_{j} B_{i j} .
$$

Here, the sums are over the $N_{c}$ components of the mixture, $x_{i}$ is the mole fraction of component $i$, and $B_{i j}$ can be expressed in terms of the pair potentials $V_{i j}(r)$ as

$$
B_{i j}=2 \pi \int_{0}^{\infty}\left[1-e^{-\beta V_{i j}(r)}\right] r^{2} d r
$$

where $\beta^{-1}=k_{\mathrm{B}} T$. Using Eq. (2) to eliminate $\rho$ from Eq. (1) and rearranging leads to

$$
\frac{D_{i}}{\left(B+T \frac{d B}{d T}\right)\left[\rho D_{i}\right]_{0}}=\frac{1}{-s^{\mathrm{ex}} / k_{\mathrm{B}}},
$$

which again is valid only in the $\rho \rightarrow 0$ limit. We identify the dimensionless quantity on the left-hand side, which is clearly a function of $s^{\text {ex }}$ only at low density, as the generalized Rosenfeld reduced form of the tracer diffusivity, $D_{i}^{\mathrm{GR}}$,

$$
D_{i}^{\mathrm{GR}} \equiv \frac{D_{i}}{\left(B+T \frac{d B}{d T}\right)\left[\rho D_{i}\right]_{0}} .
$$

Note that the expression for $D^{\mathrm{GR}}$, the generalized Rosenfeld self-diffusion coefficient for a monatomic fluid, is obtained by replacing $D_{i}$ with $D$ in Eq. (5).

Although the definition for $D_{i}^{\mathrm{GR}}$ given in Eq. (5) is compact, it is more convenient for making predictions for model systems if $\left[\rho D_{i}\right]_{0}$ is further expressed in terms of the mole fractions of the species, the associated pair potentials, the particle masses, and the temperature. Below, we present simple theoretical expressions for carrying this out for particles with hard-sphere (HS) and soft (continuous) interactions, respectively.

\section{A. Hard-particle interactions}

For models with hard-sphere interactions, an expression for $\left[\rho D_{i}\right]_{0}$ is easily obtained within Enskog kinetic theory $[41,42]$ In particular, the product $\rho D_{i}$ is given by

$$
\rho D_{i}=\frac{3}{8 \pi^{1 / 2}} \frac{\sqrt{k_{\mathrm{B}} T / m_{i}}}{\sum_{j=1}^{N_{c}} x_{j} \sigma_{i j}^{2} g\left(\sigma_{i j}^{+}\right)\left[\frac{1}{2}\left(1+\frac{m_{i}}{m_{j}}\right)\right]^{-1 / 2}},
$$

where $m_{i}$ is the mass of component $i, \sigma_{i j}$ is the hard-sphere contact diameter between particles of type $i$ and $j$, and $g_{i j}\left(\sigma_{i j}^{+}\right)$is the value of the radial distribution function between particles of type $i$ and $j$ at contact. The low-density limit $\left[\rho D_{i}\right]_{0}$ is obtained by substituting $g_{i j}\left(\sigma_{i j}^{+}\right)=1$ into Eq. (6), which gives

$$
\left[\rho D_{i}\right]_{0}=\frac{3}{8 \pi^{1 / 2}} \frac{\sqrt{k_{\mathrm{B}} T / m_{i}}}{\sum_{j=1}^{N_{c}} x_{j} \sigma_{i j}^{2}\left[\frac{1}{2}\left(1+\frac{m_{i}}{m_{j}}\right)\right]^{-1 / 2}} .
$$

When computing $D_{i}^{\mathrm{GR}}$ for the hard-sphere and WidomRowlinson model mixtures discussed in Sec. III, we simply substitute Eq. (7) into Eq. (5).

\section{B. Soft-particle interactions}

We also study fluids of soft particles in this work, i.e., particles with continuous and bounded interactions that cannot be treated as hard spheres with an effective temperaturedependent diameter. In order to predict $\left[\rho D_{i}\right]_{0}$ for these models, we use an approximate theory due to Tankeshwar and co-workers $[43,44]$, which we refer to as the Tankeshwar diffusion model (TDM). We have found that this basic theoretical approach strikes a reasonable balance between simplicity and accuracy. It has been shown to semiquantitatively describe how temperature, composition, and density affect the tracer diffusivity of Lennard-Jones fluids, the onecomponent plasma, and Yukawa fluids [45]. We have also found that it approximately captures how temperature and density affect the diffusion coefficient of the Gaussian-core fluid. 
The details of the TDM are discussed extensively elsewhere [43-45]. In short, it is based on an approximate expression for the velocity autocorrelation function and hence the tracer diffusivity $D_{i}$ via the Green-Kubo relation, for each component $i$ of the fluid in terms of two parameters: the Einstein frequency $\omega_{i}$ and a "jumping" frequency $\tau_{i}$. The values of these parameters are obtained by ensuring that the velocity autocorrelation functions satisfy some exact microscopic sum rules.

Within this model, the product $\rho D_{i}$ is given by

$$
\rho D_{i}=\rho \frac{k_{\mathrm{B}} T}{m_{i}} \frac{\pi}{2} \tau_{i} \sec \left(\frac{\pi}{2} \omega_{i} \tau_{i}\right),
$$

where

$$
\begin{gathered}
\tau_{i}^{-2}=\frac{\rho A_{i}^{(4)}-\left[\rho A_{i}^{(2)}\right]^{2}}{4 \rho A_{i}^{(2)}}, \\
\omega_{i}^{2}=-\frac{5\left[\rho A_{i}^{(2)}\right]^{2}-\rho A_{i}^{(4)}}{4 \rho A_{i}^{(2)}},
\end{gathered}
$$

and

$$
\begin{aligned}
A_{i}^{(2)}= & \frac{4 \pi}{3} \sum_{j=1}^{N_{c}} \frac{x_{j}}{m_{j}} \int_{0}^{\infty} d r r^{2} g_{i j}(r)\left(\frac{2}{r} \frac{d V_{i j}}{d r}+\frac{d^{2} V_{i j}}{d r^{2}}\right), \\
A_{i}^{(4)}= & \frac{4 \pi}{3} \sum_{j=1}^{N_{c}} \frac{x_{j}}{m_{i}}\left(\frac{1}{m_{i}}+\frac{1}{m_{j}}\right) \int_{0}^{\infty} d r r^{2} g_{i j}(r) \\
& \times\left\{\frac{2}{r^{2}}\left(\frac{d V_{i j}}{d r}\right)^{2}+\left(\frac{d^{2} V_{i j}}{d r^{2}}\right)^{2}\right\} .
\end{aligned}
$$

In Eq. (9), $V_{i j}$ is the pair potential between particles of species $i$ and $j$, and terms involving three-body static correlations have been omitted. In order to evaluate $\left[\rho D_{i}\right]_{0}$, we take the $\rho \rightarrow 0$ limit of Eqs. (8) and (9), which leads to

$$
\left[\rho D_{i}\right]_{0}=\frac{k_{\mathrm{B}} T}{m_{i}}\left(\frac{A_{i, 0}^{(4)}}{\left[A_{i, 0}^{(2)}\right]^{3}}\right)^{1 / 2} .
$$

Here $A_{i, 0}^{(2)} \equiv \lim _{\rho \rightarrow 0} A_{i}^{(2)}$ and $A_{i, 0}^{(4)} \equiv \lim _{\rho \rightarrow 0} A_{i}^{(4)}$, each of which follow by replacing $g_{i j}(r)$ in Eq. (9), with the Boltzmann factor of the pair potential,

$$
\lim _{\rho \rightarrow 0} g_{i j}(r)=\exp \left[-\beta V_{i j}(r)\right] .
$$

When computing $D_{i}^{\mathrm{GR}}$ for the Gaussian-core mixtures discussed in Sec. III, we substitute Eq. (11) into Eq. (5).

\section{MODEL SYSTEMS AND SIMULATION METHODS}

As discussed in Sec. I, a key aim of this paper is to investigate whether it is possible to construct an excessentropy-based strategy for predicting tracer diffusivity generic enough to be successfully applied to fluid mixtures with either hard (impenetrable) or soft (penetrable) interparticle interactions. For our model systems, we choose familiar representations for both: the HS pair potential for the former and the Gaussian-core pair potential for the latter.

The HS pair potential is discontinuous and athermal, assigning infinite energy to configurations that have particle overlaps and zero energy to all others. It is thus represented as

$$
V_{i j}^{\mathrm{HS}}(r)= \begin{cases}\infty & r<\sigma_{i j} \\ 0 & r \geq \sigma_{i j} .\end{cases}
$$

Here, $\sigma_{i j}$ is the contact diameter between particles of type $i$ and $j$. We investigate several binary HS mixtures with additive diameters, i.e., $\sigma_{i j}=\left(\sigma_{i}+\sigma_{j}\right) / 2$. In particular, we first study compositional effects on tracer diffusivity and excess entropy using a system composed of equimass $\left(m_{1} / m_{2}=1\right)$ particles with diameter ratio $\sigma_{1} / \sigma_{2}=1.3$. For this system, we examine mole fractions of component one in the range 0.1 $\leq x_{1} \leq 0.9$. We also investigate the effects of diameter ratio by considering particles with $\sigma_{1} / \sigma_{2}=1.3,2.0,3.0,5.0$. These latter studies are carried out at fixed composition $x_{1}=0.1$. All of the above systems are studied across a wide range of packing fractions $\varphi=\pi\left(x_{1} \sigma_{1}^{3}+x_{2} \sigma_{2}^{3}\right) / 6$ spanning between 0.05 (dilute gas) and 0.5 [near the single-component (SC) HS freezing transition].

We also consider a highly nonadditive version of the binary HS mixture: the Widom-Rowlinson (WR) model [46]. In this system, the contact diameter between particles of the same type is zero $\left(\sigma_{11}=\sigma_{22}=0\right)$, but the cross diameter is finite $\sigma_{12}=\sigma_{21}=\sigma$ and $m_{1}=m_{2}$. As might be imagined, this system exhibits entropically driven phase separation at sufficiently high density, which we avoid here by studying 0 $<\rho \sigma^{3}<0.7$. Since the model is symmetric with respect to the interactions, we can deduce global behavior by studying mole fractions in the range $0<x_{1} \leq 0.5$.

Finally, we study fluids composed of soft particles that interact via the bounded Gaussian-core pair potential [47] given by

$$
V_{i j}^{\mathrm{GC}}(r)=\epsilon_{i j} \exp \left[-\left(r / \sigma_{i j}\right)^{2}\right]
$$

where $\epsilon_{i j}$ and $\sigma_{i j}$ are parameters that characterize the energy and length scale, respectively, of the interaction between particles of type $i$ and $j$. For the simulations in this work, we truncate the interparticle interactions at a separation of $3.2 \sigma_{i j}$. We examine both single-component and twocomponent Gaussian-core fluids. For the latter, we adopt the same parameters used in a previous investigation of the static structure and thermodynamics of that system [48]. Specifically, we assign $\sigma_{22}=0.665 \sigma_{11}$ and $\sigma_{12}=\sqrt{\left(\sigma_{11}^{2}+\sigma_{22}^{2}\right) / 2}, \epsilon_{11}$ $=\epsilon_{22}$ and $\epsilon_{12}=0.944 \epsilon_{11}$ (which encourages mixing), and $m_{1}$ $=m_{2}$. We investigate the binary Gaussian-core fluid at compositions $0.1 \leq x_{1} \leq 0.9$, temperatures $0.05 \leq k_{\mathrm{B}} T / \epsilon_{11} \leq 0.4$, and densities $0.05 \leq \rho \sigma_{11}^{3} \leq 1.0$. At some temperatures, the maximum density in this range is not an isotropic fluid due to propensity of the system to phase separate. We excluded from our analysis any state points that showed thermodynamic or structural indications of phase separation.

To explore the dynamic properties of the above systems, we perform molecular-dynamics (MD) simulations. For the HS and WR mixtures, we use a standard event-driven algorithm [49]. For the binary Gaussian-core fluid, the equations 
of motion are integrated using the velocity-Verlet method [50] with time step $\delta t=0.05 \sqrt{m_{1} \sigma_{11}^{2} / \epsilon_{11}}$. All MD simulations are carried out in the microcanonical ensemble with $N$ =3000-5000 particle using a periodically replicated cubic simulation cell with volume $V$, chosen in accord with the desired fluid density. Tracer diffusion coefficients $D_{i}$ are calculated by fitting the long time average mean-squared displacement of the $i$-type particles $\left\langle\delta r_{i}^{2}\right\rangle$ to the Einstein relation $6 D_{i} t=\left\langle\delta r_{i}^{2}\right\rangle$. Note that, for the case of the monatomic fluid, this definition of the tracer diffusivity reduces to the selfdiffusivity. We perform multiple-independent simulations at several state points for each model, and we find the relative standard error in tracer diffusivities to be less than $1 \%$.

Thermodynamic properties of the Gaussian-core fluid mixtures are computed using grand-canonical transitionmatrix Monte Carlo (GC-TMMC) simulations. These simulations are conceptually equivalent to a series of semigrand simulations performed over a range of fluid densities stitched together using ghost insertion or deletion moves. Details of this method can be found elsewhere $[51,52]$. These simulations require fixed values of the activity, $\left\{\xi_{1}, \xi_{2}\right\}$, volume, $V$, and temperature, $T$, as inputs. The activity is defined as $\xi_{i}=\Lambda_{i}^{-3} \exp \left(\mu_{i} / k_{\mathrm{B}} T\right)$, where $\mu_{i}$ and $\Lambda_{i}$ are the chemical potential and the thermal de Broglie wavelength of component $i$, respectively. All GC-TMMC simulations for the Gaussian-core fluid mixtures reported here use a system volume of $V=343$. For the activities, the values of $\ln \xi_{i}$ that we use span from 32.63 at the lowest temperature to 12.24 at the highest temperature investigated. Thermodynamic properties at other values of activity are obtained via the histogram reweighting technique. The primary quantity obtained from GC-TMMC is the particle number probability distribution $\Pi\left(N ;\left\{\mu_{i}\right\}, V, T\right)$. From this, excess entropies are trivially calculated (see [10]). System size effects in excess entropy for the Gaussian-core mixtures are found to be negligible by comparing results to a series of simulations using a smaller volume of $V=216$. We confirmed that the equation of state (and hence excess entropy) of Gaussian-core fluid mixtures produced from the GC-TMMC simulations is statistically indistinguishable with that produced from molecular-dynamics simulations.

GC-TMMC calculations for the WR mixtures are performed using a system volume of $V=343$. For this fluid, simulations are completed with activity values of $\xi_{1}=\xi_{2}=1$, and histogram reweighting is applied to obtain thermodynamic quantities at other values of activity. System size effects are examined by performing simulations over a limited density and composition range with a volume of $V=1000$ and are also found to be negligible.

The excess entropy data we present for the binary HS mixtures is calculated from the accurate Boublik-MansooriCarnahan-Starling-Leland (BMCSL) equation of state $[53,54]$. As a check, we compared the BMCSL values for compressibility factor and excess entropy against those obtained via molecular-dynamics simulations for selected state points as a function of particle diameter ratio and packing fraction, and we found the agreement to be excellent.

Finally, we also compare the results for the binary systems to corresponding single-component systems. For the single-component HS system we use the data of [11] and for the single-component Gaussian-core fluid we use the data of [30].

\section{RESULTS AND DISCUSSION}

\section{A. Hard-sphere mixtures}

\section{Compositional effects}

We begin by investigating the effects of composition on mixtures of $\mathrm{HS}$ particles with size ratio $\sigma_{1} / \sigma_{2}=1.3$. Figure 1(a) displays the tracer diffusion coefficients $D_{i}$ of the two components as a function of total packing fraction $\varphi$ for several different compositions, indicated by the mole fraction of large particles, $x_{1}$. As must be the case, when one of the species is present in high concentration, its tracer diffusivity approaches the value of the self-diffusion coefficient $D$ of the single-component HS fluid at the same packing fraction $\varphi$. However, the tracer diffusivity of the dilute component is generally different than $D$. In particular, when component 1 (the larger particles) is dilute, one should expect $D_{1}<D$. This logic can be qualitatively rationalized by the fact that, on average, motion of the larger solute would require larger local structural rearrangements (i.e., fluctuations) than for the motion of the smaller solvent particles. Conversely, by an analogous argument, one expects $D_{2}>D$ if component 2 (the smaller particles) is dilute. The data in Fig. 1(a) are consistent with these expectations.

It is interesting to note that the compositional variation in $\ln D_{i}$ is fairly insensitive to the value of $\varphi$. Moreover, the excess entropy [Fig. 1(n)] exhibits almost no compositional dependence whatsoever, and its packing fraction dependence for any particular composition is nearly identical to that of the single-component HS fluid. All of this suggests that an appropriate composition-dependent rescaling of the tracer diffusivity data might (approximately) make it a singlevalued function of excess entropy. Indeed, Figs. 1(c) and 1(d) show that the generalized Rosenfeld tracer diffusivities $D_{i}^{\mathrm{GR}}$ of Eq. (5) for both species collapse onto a single curve (that describing the $\mathrm{SC}$ fluid data, $D_{\mathrm{SC}}^{\mathrm{GR}}$ ) when plotted versus excess entropy, while no data collapse occurs if the tracer diffusivities are naïvely represented in the original Rosenfeld reduced form $D_{i}^{\mathrm{R}}$.

The single-component relationship $D_{\mathrm{SC}}^{\mathrm{GR}}\left(s^{\mathrm{ex}}\right)$ can be described by a piecewise function. The form of its low-density (low $-s^{\mathrm{ex}}$ ) scaling is an inverse power law given by Eq. (4). From Fig. 1(d), we infer that when $-s^{\mathrm{ex}} / k_{\mathrm{B}} \gtrsim 1$, the relationship becomes approximately exponential. A least-squares fit assuming these generic function forms, i.e.,

$$
D_{\mathrm{SC}}^{\mathrm{GR}}\left(s^{\mathrm{ex}}\right)= \begin{cases}\alpha\left[-s^{\mathrm{ex}} / k_{\mathrm{B}}\right]^{-1} & -s^{\mathrm{ex}} / k_{\mathrm{B}}<1 \\ A \exp \left[-B s^{\mathrm{ex}} / k_{\mathrm{B}}\right] & -s^{\mathrm{ex}} / k_{\mathrm{B}}>1,\end{cases}
$$

yields $\alpha=0.95, A=1.85$, and $\beta=0.74$ and describes the simulation data very well (see red dashed line in Fig. 1).

The data collapse of Fig. 1(d) suggests that tracer diffusivities, $D_{1}$ and $D_{2}$, of this mixture might also be adequately predicted using Eq. (15) together with knowledge of the pair potentials, composition, and excess entropy of the mixture. Specifically, the generalized Rosenfeld scaling prediction for tracer diffusivity of component $i$ is given by 

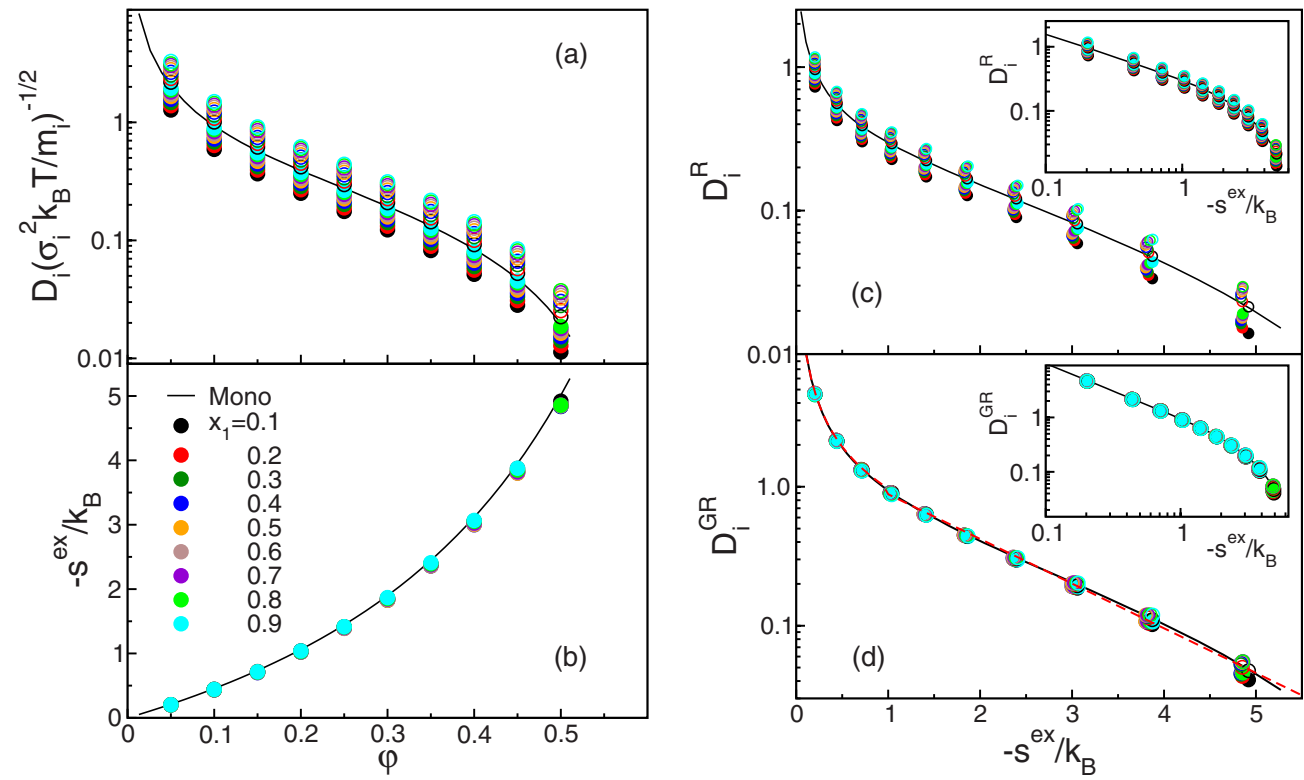

FIG. 1. (Color online) Properties of the binary HS mixture with particle diameter ratio $\left(\sigma_{1} / \sigma_{2}\right)=1.3$, equal mass, and a variety of compositions. (a) Tracer diffusion coefficients $D_{i}$ and (b) (negative) excess entropy $-s^{\mathrm{ex}}$ as a function of packing fraction $\varphi$. (c) Rosenfeld $D_{i}^{\mathrm{R}}$ and (d) generalized Rosenfeld $D_{i}^{\mathrm{GR}}$ tracer diffusivities as a function of $-s^{\mathrm{ex}}$. Filled and open symbols denote component 1 (large) and 2 (small), respectively. The color of symbols denotes the mole fraction of component $1, x_{1}$, specified in the legend of (a). The solid line in each figure is the result for the single-component HS system. The dashed red line in (d) represents a least-squares fit of the data to Eq. (15), which results in $\alpha=0.95, A=1.85$, and $\beta=0.74$. In (c) and (d), the insets are the same as the main plots but on a log-log scale.

$$
D_{i}^{\text {predicted }}\left(s^{\mathrm{ex}}\right)=\left\{\left[\rho D_{i}\right]_{0}\left[B+T \frac{d B}{d T}\right]\right\} D_{\mathrm{SC}}^{\mathrm{GR}}\left(s^{\mathrm{ex}}\right),
$$

with $B$ from Eq. (2), $\left[\rho D_{i}\right]_{0}$ from Eq. (7), and $D_{\mathrm{SC}}^{\mathrm{GR}}\left(s^{\mathrm{ex}}\right)$ from the fit of the single-component data to Eq. (15).

One way to quantitatively assess the relative predictive ability of Eq. (16) is to compare it to the results of, e.g., the basic Enskog theory given by Eq. (6). Both equations require as inputs several pieces of information, including the form of the pair potentials and the mixture composition. While predictions based on the generalized Rosenfeld scaling also require knowledge of $s^{\mathrm{ex}}$ for the mixture and properties of the single-component system, Enskog theory requires knowledge of the state-dependent contact values of the three partial radial distribution functions of the mixture.

Figure 2 displays the relative error in the predicted tracer diffusivity to the simulated diffusivity $D_{i}^{\text {predicted }} / D_{i}$. Enskog theory [Fig. 2(a)] provides good predictions at high values of tracer diffusivity (i.e., low $\varphi$ ). However, as the value of $D_{i}$ decreases (i.e., $\varphi$ increases) Enskog theory first underpredicts, then ultimately significantly overpredicts $D_{i}$. When looking at the entire range of $\varphi$ studied here, $80 \%$ of the Enskog theory predictions lie within $20 \%$ of the molecular simulation data. On the other hand, the excess entropy based expression of Eq. (16) [Fig. 2(b)] predicts the tracer diffusivities semiquantitatively for all state points investigated here $(100 \%$ of predictions within $20 \%$ of the simulation data).

\section{Particle-size asymmetry effects}

We also study the effects of particle-size asymmetry on the relationship between excess entropy and tracer diffusivity by examining a series of binary HS mixtures at composition $x_{1}=0.1$ and packing fractions in the range $0<\varphi \leq 0.5$. Particles of types 1 and 2 were taken to have identical masses, but we investigated several systems with different diameter ratios $\left(\sigma_{1} / \sigma_{2}=1.3,2.0,3.0\right.$, and 5.0). Figure 3(a) displays the tracer diffusivities, $D_{1}$ and $D_{2}$, for these systems. Increasing the magnitude of the diameter ratio leads to progressively larger deviation of the tracer diffusivities from the selfdiffusion coefficient $D$ of the HS fluid at the same $\varphi$. As expected, larger particles diffuse slower than smaller particles $\left[D_{1}<D<D_{2}\right]$. How is the excess entropy affected by increasing the ratio of particle diameters? Figure 3(b) shows that increasing $\sigma_{1} / \sigma_{2}$ at fixed $\varphi$ and $x_{1}$ systematically de-

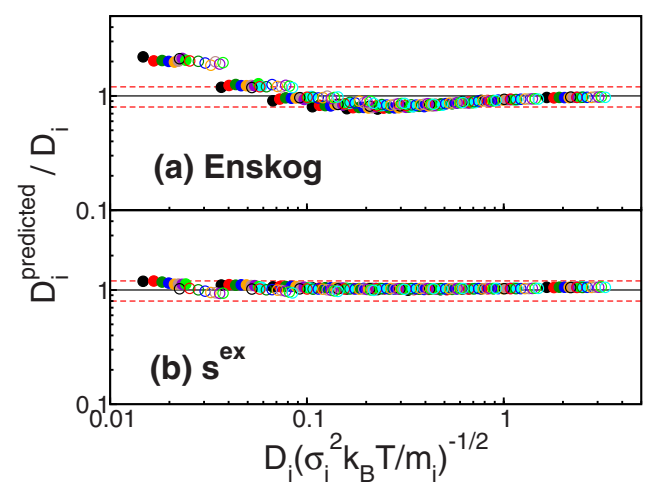

FIG. 2. (Color online) Ratio of tracer diffusivity predicted from (a) Enskog theory [Eq. (6)] and (b) from excess entropy and the single-component HS result (generalized Rosenfeld scaling) [Eq. (16)] for a HS mixture with $\left(\sigma_{1} / \sigma_{2}\right)=1.3$, equal mass, and a variety of compositions. Red dashed lines represent $20 \%$ relative error of prediction. Symbols have the same meaning as in Fig. 1. 

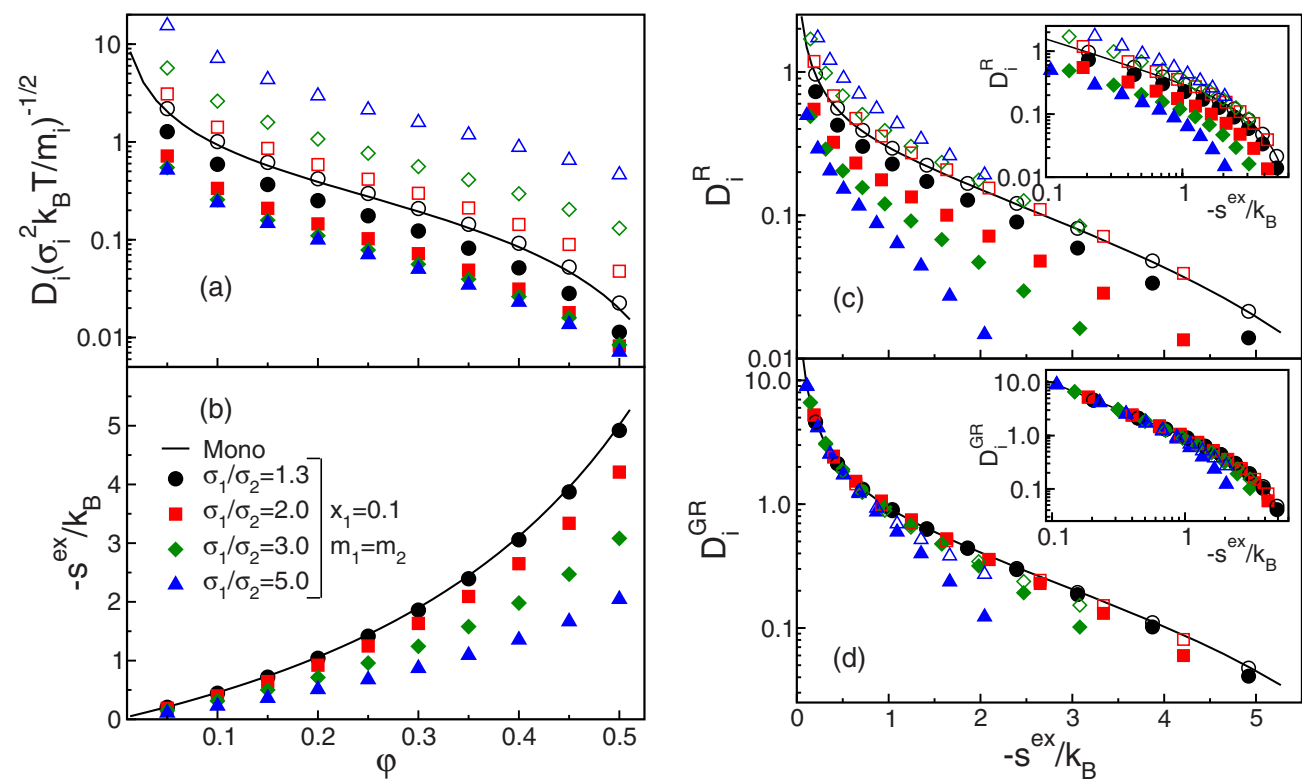

FIG. 3. (Color online) Properties of the binary HS mixture at composition $x_{1}=0.1$, equal mass, and several size ratios $\left(\sigma_{1} / \sigma_{2}\right)$ [see legend in (a)]. (a) Tracer diffusion coefficient $D_{i}$ and (b) (negative) excess entropy $-s^{\text {ex }}$ versus packing fraction $\varphi$. (c) Tracer diffusivity reduced in the original Rosenfeld $D_{i}^{\mathrm{R}}$ and (d) generalized Rosenfeld $D_{i}^{\mathrm{GR}}$ forms as a function of $-s^{\mathrm{ex}}$. Filled and open symbols represent large (component 1) and small (component 2) spheres. Symbol shapes denote different particle diameter ratios, as described in the legend of (a). The solid line in each figure is the result for the single-component HS system. In (c) and (d) the insets are the same as the main plots but on a log-log scale.

creases $-S^{\mathrm{ex}}$ (i.e., weakens the static interparticle correlations). This effect is qualitatively connected to the more efficient packing arrangements that spheres can sample when significant polydispersity is present $[55,56]$.

Given that increasing size ratio uniformly reduces structural correlations but impacts the dynamics of large and small particles in different ways, it might not be surprising that tracer diffusivity data represented in the original Rosenfeld form, $D_{i}^{\mathrm{R}}$, does not collapse when plotted versus excess entropy [Fig. 3(c)]. The $D_{i}^{\mathrm{R}}$ data for the smallest size ratio $\sigma_{1} / \sigma_{2}=1.3$ are qualitatively similar to the single-component result. However, there is significant deviation for larger diameter ratios, with excess entropy underpredicting the mobility of smaller particles and overpredicting that of larger particles. Figure 3(d) shows, however, that tracer diffusivity reduced in the generalized Rosenfeld form, $D_{i}^{\mathrm{GR}}$, mostly collapses to the single-component curve when plotted versus $-s^{\mathrm{ex}}$. The most pronounced deviations are for the largest size ratio $\left(\sigma_{1} / \sigma_{2}=3,5\right)$ at the highest packing fractions $(\phi$ $\geq 0.45)$.

Figure 4 quantitatively compares the predictions of Enskog theory [Eq. (6)] with those based on the generalized Rosenfeld scaling [Eq. (16)]. At high values of $D_{i}$ (low $\varphi$ ), both methods provide accurate predictions. As before, for decreasing $D_{i}$ (increasing $\varphi$ ), Enskog theory first underpredicts and then ultimately overpredicts the tracer diffusivities. The excess entropy based predictions never underpredict but eventually overpredict the mobility at high values of $\varphi$. As an overall measure, the Enskog and the excess entropy expressions predict $70 \%$ and $80 \%$ of the tracer diffusivities within $20 \%$ of the simulated values, respectively. Moreover, we note that while the excess entropy method predicts the tracer diffusivities of the two components with similar reliability, the Enskog expression does well for the small particles $(90 \%$ within $20 \%$ ), but poorly for the large particles (50\% within $20 \%)$.

\section{Two-body excess entropy scaling}

As noted in Sec. I, an alternative excess entropy based scaling for diffusion was introduced by [3], who found that an appropriately reduced form of the self-diffusion coefficient $D^{\mathrm{Z}}$ for atomic fluids at moderate densities is nearly a universal function of the two-body contribution to the excess

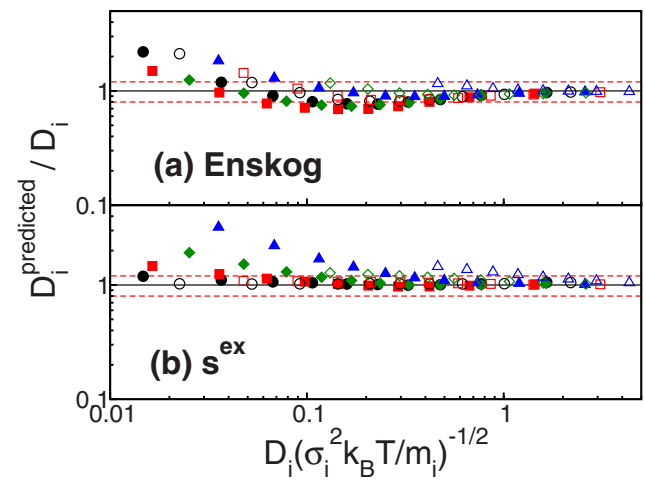

FIG. 4. (Color online) Ratio of tracer diffusivity predicted from (a) Enskog theory [Eq. (6)] and (b) from excess entropy and the single-component HS data (i.e., generalized Rosenfeld scaling) [Eq. (16)], for a HS mixture with equal mass, composition $x_{1}=0.1$, and a variety of size ratios $\left(\sigma_{1} / \sigma_{2}\right)$. Red dashed lines represent $20 \%$ relative error of prediction. Symbols have the same meaning as in Fig. 3. 


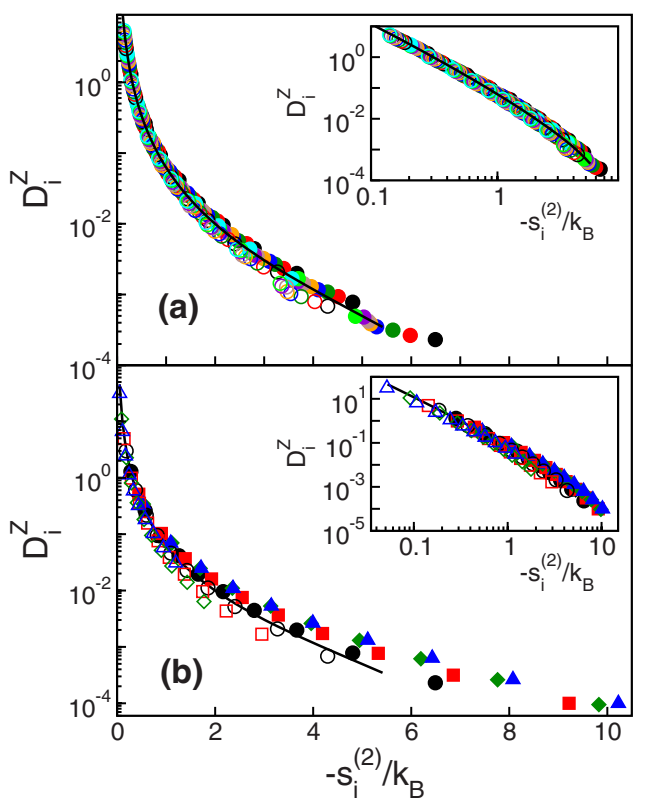

FIG. 5. (Color online) Tracer diffusivity reduced in generalized Dzugutov form $D_{i}^{\mathrm{Z}}$ discussed in text versus (negative) $i$-component contribution to two-body excess entropy $-s_{i}^{(2)}$ of binary HS mixtures. (a) Particle diameter ratio $\sigma_{1} / \sigma_{2}=1.3$, equal mass, and a variety of compositions. Symbols have same meaning as Fig. 1. (b) Composition $x_{1}=0.1$, equal mass, and a variety of size ratios. Symbols have same meaning as Fig. 3. Insets are the same as the main plots but with a log-log scale.

entropy $s^{(2)}$. Subsequently, others have suggested a generalization of this scaling [39] to predict tracer diffusivities of fluid mixtures. In the generalization, the reduced tracerdiffusion coefficient defined as $D_{i}^{\mathrm{Z}} \equiv D_{i} / \chi_{i}$, where

$$
\chi_{i} \equiv 4\left(\pi k_{\mathrm{B}} T\right)^{1 / 2} \sum_{j=1}^{N_{\mathrm{c}}} x_{i} \rho \sigma_{i j}^{4} g_{i j}\left(\sigma_{i j}^{+}\right)\left(\frac{m_{i}+m_{j}}{2 m_{i} m_{j}}\right)^{1 / 2}
$$

is thought to approximately scale with the $i$-component contribution to the two-body excess entropy defined as [39]

$$
s_{i}^{(2)} / k_{\mathrm{B}} \equiv-\frac{1}{2} \rho \sum_{j=1}^{N_{\mathrm{c}}} x_{j} \int d \mathbf{r}\left\{g_{i j}(\mathbf{r}) \ln g_{i j}(\mathbf{r})-\left[g_{i j}(\mathbf{r})-1\right]\right\} .
$$

Note that the two-body excess entropy per particle is given by $s^{(2)}=\sum_{i} x_{i} s_{i}^{(2)}[57]$.

In Figs. 5(a) and 5(b) we examine the mixture generalization of the Dzugutov scaling for the HS systems discussed above. Specifically, we show data with fixed diameter ratio and varying composition in panel (a) and fixed composition and varying diameter ratio in panel (b). Both sets of data more or less track the scaling. However, deviations from the single component curve appear systematic. The singlecomponent relation with $s_{i}^{(2)}$ overpredict the small sphere mobility and underpredict the large sphere mobility.

Unfortunately, the predictive value of this type of scaling is inherently limited by the fact that the single-component data cannot access the large values of $-s_{i}^{(2)}$ realized by the large spheres in a mixture. The former reach a value of $5.5 k_{\mathrm{B}}$ at $\varphi=0.5$, while the latter are greater than $10 k_{\mathrm{B}}$ for the largest size ratios examined here. As a result, "predicting" tracer diffusivities of a mixture would require some systematic way of extrapolating the single-component curve by a substantial amount. As discussed in Sec. I, the scaling is also limited to systems, such as the HS fluid, for which the interparticle repulsions are steep enough to define an effective hard-core diameter to each interaction. Thus, it will be of little use for studying systems with bounded interactions such as the Gaussian-core potential or a other models that characterize the soft effective interactions between macromolecular or supramolecular species in solution [37].

\section{B. Widom-Rowlinson mixtures}

Here we examine the behavior of the WR model fluid introduced in Sec. III. Recall that it is defined as a mixture of nonadditive hard spheres with $\sigma_{11}=\sigma_{22}=0$ but $\sigma_{12}=\sigma$. Figure 6(a) displays the tracer diffusivity $D_{i}$ as a function of density for a several compositions $x_{1}$. Note that the $D_{i}$ is always greater then the self-diffusion of the single-component HS fluid since the number of collisions per unit time will clearly be less in the WR fluid than in the HS fluid at the same density. At $x_{1}=0.5, D_{1}=D_{2}$, since the fluid is symmetric. At fixed density, as $x_{1}$ decreases, $D_{1}$ decreases while $D_{2}$ increases. This is because the dilute species will experience many more collisions per unit time (it has more neighbors of the opposite type) than the concentrated species. Likewise, Fig. 6(b) shows $-s^{\mathrm{ex}}$ for the WR fluids is always less than that of a single-component HS fluid of the same density. This is expected since particles of the same type do not directly exclude volume from one another, which in turn reduces the entropic driving force for forming strong interparticle correlations. Decreasing $x_{1}$ from 0.5 toward zero at fixed density decreases $-s^{\mathrm{ex}}$ because it increases the number of particles in the system that do not interact.

As was the case for the HS fluid mixtures, Fig. 6(c) shows that the tracer diffusivity reduced in the original Rosenfeld form, $D_{i}^{\mathrm{R}}$, is not even approximately a single-valued function of $s^{\mathrm{ex}}$. On the other hand, Fig. 6(d) shows that the tracer diffusivity data cast in the generalized Rosenfeld form, $D_{i}^{\mathrm{GR}}$, largely collapses when plotted versus excess entropy. Interestingly, $D_{i}^{\mathrm{GR}}$ of the WR fluid is well described by the mathematical form of the single-component HS data. The quality of the collapse is more easily seen on a log-log scale [inset to Fig. 6(d)].

Figures 7(a) and 7(b) compare the accuracy of predicting tracer diffusivity of the components of the WR fluid based on Enskog theory [Eq. (6)] versus excess entropy of the WR mixture and the single-component relation for the HS fluid [Eq. (16)]. As for the HS fluid, Enskog theory predicts $80 \%$ of the WR data within $20 \%$ of the simulation values. In contrast, the excess entropy method predicts $97 \%$ of the data within $20 \%$ of the simulated tracer diffusivities.

Lastly, since the WR model is composed of (nonadditive) hard particles, it represents another good test case for the mixture generalization of the Dzugutov scaling. Figure 8, however, clearly shows that this two-body scaling does not 

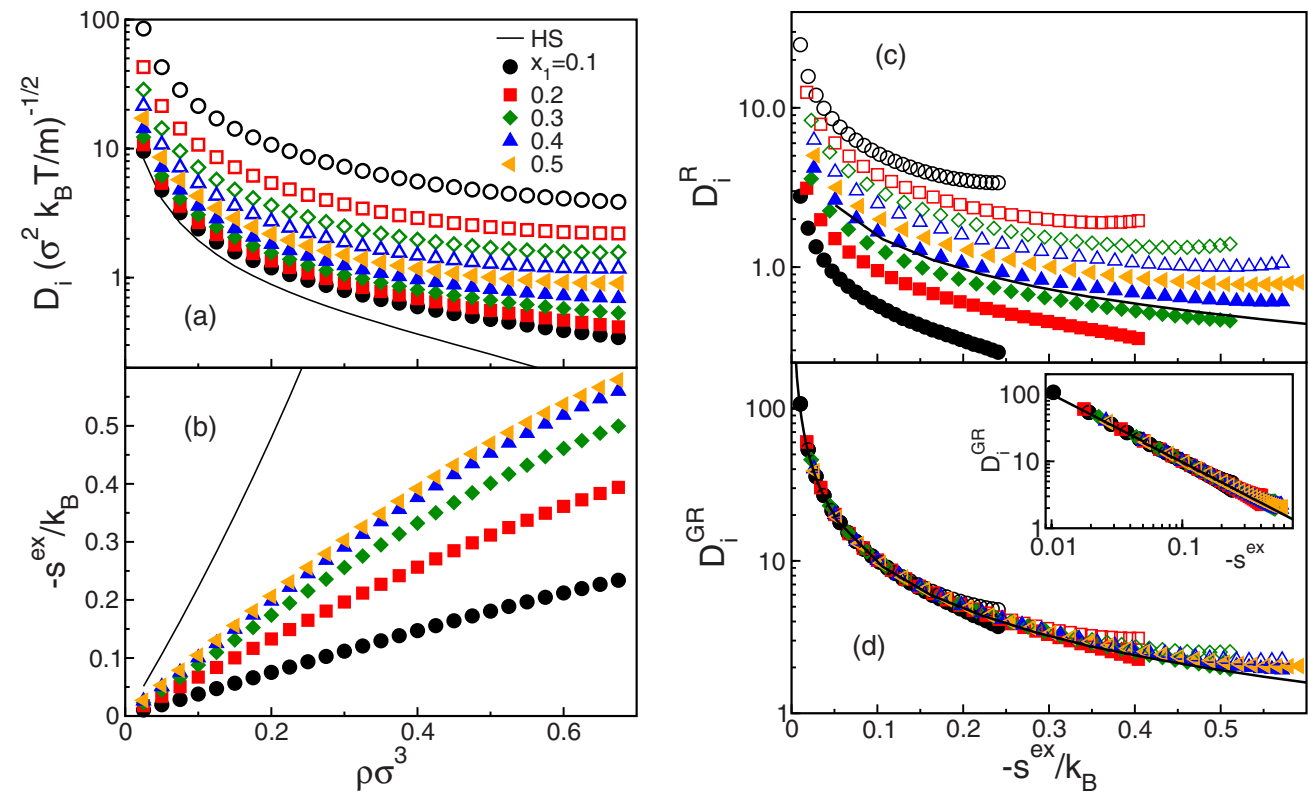

FIG. 6. (Color online) Properties of the Widom-Rowlinson mixture. (a) Tracer diffusivities $D_{i}(i=1,2)$ and (b) (negative) excess entropy $-s^{\mathrm{ex}}$ as function of density $\rho \sigma^{3}$. (c) Tracer diffusivity reduced in original Rosenfeld form $D_{i}^{\mathrm{R}}$ and (d) generalized Rosenfeld form $D_{i}^{\mathrm{GR}}$ as a function of $-s^{\mathrm{ex}}$. Filled and open symbols denote component 1 and 2, respectively. The symbol type denotes the mole fraction of component $1, x_{1}$, indicated in the legend of (a). The solid line in each figure is the result for the single-component HS fluid. In (d) the insets are the same as the main plots but on a log-log scale.

collapse the WR data. In general, particles of type $i$ diffuse considerably faster than would be predicted based on the single-component HS fluid behavior and the $i$ component of the two-body excess entropy in the WR mixture. Moreover, the magnitude of the under prediction depends sensitively on composition. This breakdown of the mixture version of the Dzugutov relation for nonadditive HS fluids indicates that it is not as widely applicable even within the limited class of HS model fluids as the generalized Rosenfeld scaling introduced here.

\section{Single-component Gaussian-core fluid}

As discussed in Sec. I, and much more extensively in $[30,31,58,59]$, the properties of the single-component

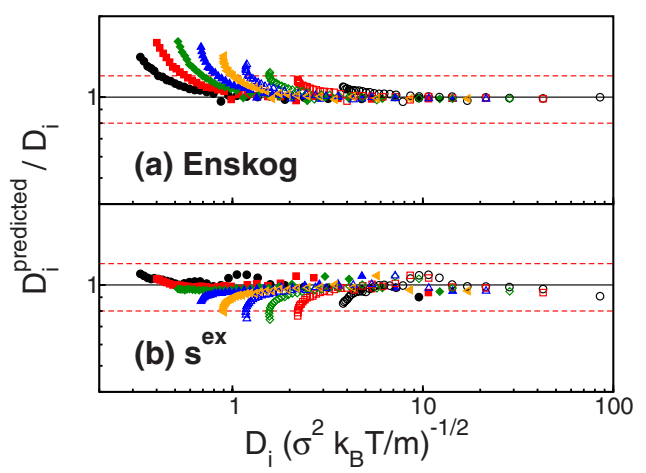

FIG. 7. (Color online) Ratio of tracer diffusivity predicted from (a) Enskog theory [Eq. (6)] and (b) from excess entropy and the single-component HS result (generalized Rosenfeld scaling) [Eq. (16)], for the Widom-Rowlinson mixture. Red dashed lines indicate $20 \%$ relative error of prediction. Symbols have the same meaning as in Fig. 6.
Gaussian-core fluid are anomalous compared to those of simple atomic liquids. For example, as shown in Fig. 9(a), the self-diffusivity $D$ of the Gaussian-core fluid first decreases and then anomalously increases as a function of particle density along an isotherm. Likewise, $-s^{\mathrm{ex}}$ at constant temperature first increases (the fluid becomes more structured) and then anomalously decreases (the fluid becomes less structured) as a function of density.

In brief, these unusual trends can be qualitatively rationalized based on the Gaussian form of the repulsion. When the density and temperature are sufficiently low, the distance between particles is larger than the range of the potential. Under these conditions, the part of the interaction that the particles sample when they "collide" appears steeply repulsive, and thus the effects of density on structure and dynamics are

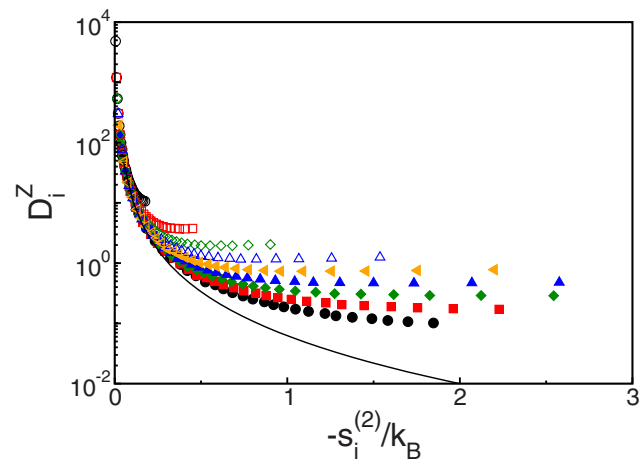

FIG. 8. (Color online) Tracer diffusivity reduced in the mixture generalized Dzugutov form discussed in text $D_{i}^{\mathrm{Z}}$ versus the (negative) $i$ component of the two-body excess entropy $s_{i}^{(2)}$ for the Widom-Rowlinson model. Symbols have same the meaning as Fig. 6. 

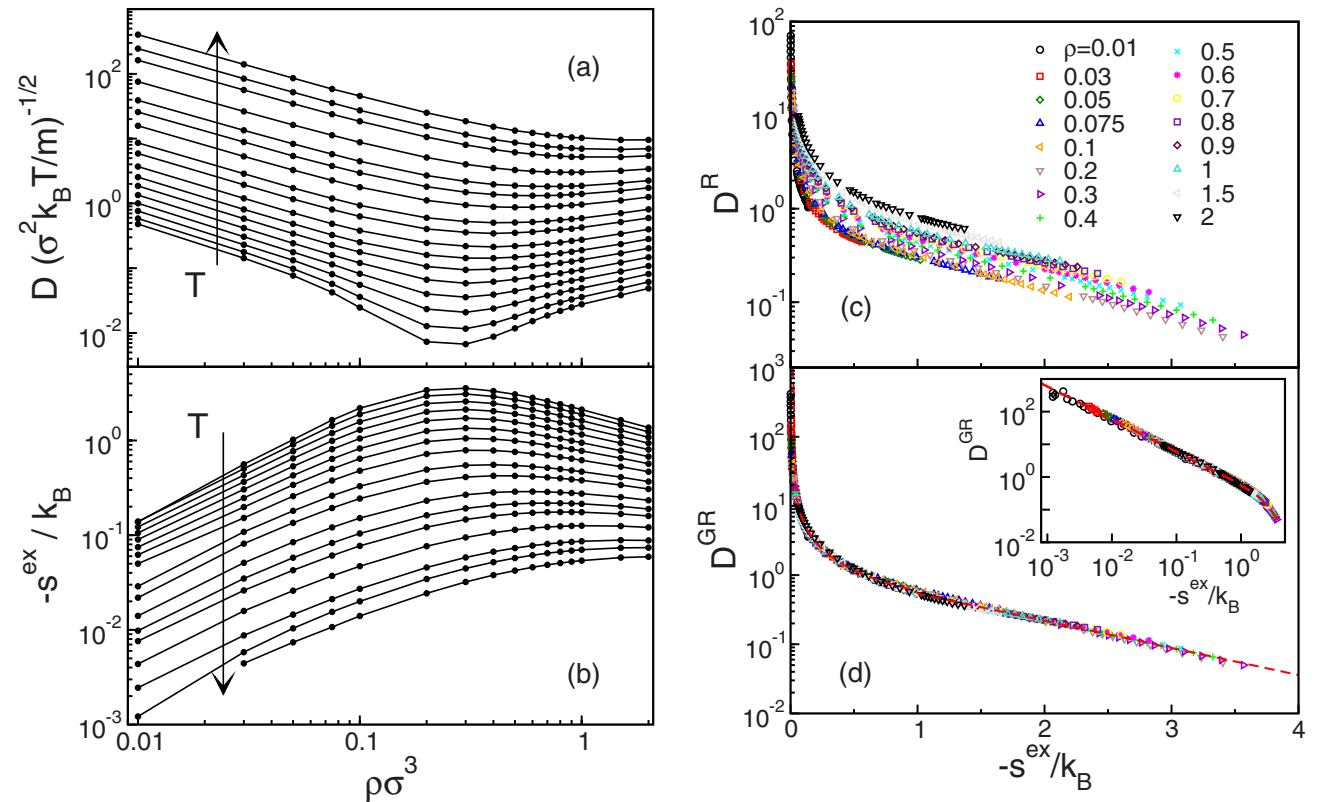

FIG. 9. (Color online) Properties of the single-component Gaussian-core fluid. (a) Self-diffusivity $D$ and (b) (negative) excess entropy $-s^{\text {ex }}$ versus density $\rho$. (c) Self-diffusivity reduced in the Rosenfeld form $D^{\mathrm{R}}$ and (d) the generalized Rosenfeld form $D^{\mathrm{GR}}$ as a function of $-s^{\text {ex }}$. In (a) and (b), arrows indicate increasing temperature $T$. In (c) and (d), symbol type corresponds to density, indicated in the legend of (c). In (d), inset is the same as the main plot but on log-log scale. In (d), the red dashed line represents a least-squares fit of the data to Eq. (15).

similar to those of HS fluid. However, at high particle densities, particles in the Gaussian-core fluid effectively overlap one another due to the bounded form of the interaction. The effect is that each particle constantly experiences largely canceling soft repulsive forces of many neighbors. Increasing the particle density under these conditions enhances this effect, paradoxically weakening the structural correlations and increasing the self-diffusivity of the fluid.

It is clear from Figs. 9(a) and 9(b) that both $D$ and $s^{\mathrm{ex}}$ are strongly correlated for the Gaussian-core fluid. In Fig. 9(c), we show the self-diffusion coefficient expressed in the original Rosenfeld form, $D^{\mathrm{R}}$, as a function of $-s^{\mathrm{ex}}$. As noted previously [30], this basic scaling is not even approximately a single-valued function of excess entropy. However, similar to the behavior of the HS and WR mixtures discussed previously, Fig. 9(d) shows that the generalized-Rosenfeld-scaled self-diffusivity $D_{i}^{\mathrm{GR}}$ collapses to a single curve when plotted versus excess entropy. The quality of the collapse at even low density is apparent in the log-log plot shown in the inset to Fig. 9(d). We also observe that the same functional form that was used to fit the single-component HS data can also be applied to the Gaussian-core system. In particular, fitting the data to Eq. (15) yields $\alpha=0.59, A=1.33$, and $B=0.90$. As shown in Fig. 9(d), Eq. (15) with these parameters (red dashed line) describes the simulation data very well.

\section{Binary Gaussian-core mixtures}

As a final test of the relationship between single-particle dynamics and excess entropy in soft-particle fluids, we examine the binary mixture of Gaussian-core particles described in Sec. III. In particular, Figs. 10(a) and 10(b) display the tracer diffusivities of the large and small Gaussian-core particles as a function of density for a variety of mixture compositions $\left(x_{1}=0.1,0.3,0.5,0.7\right.$, and 0.9$)$ and reduced temperatures $k_{\mathrm{B}} T / \epsilon=0.05,0.1$, and 0.2 . The first point of interest in the data, evident in Fig. 10(a), is that the tracer diffusivity of the larger type 1 particles displays the same anomalous trend as a function of density as the single component Gaussian-core fluid. That is, increasing the density eventually leads to an anomalous increase in $D_{1}$. However, over the density range considered here, the tracer diffusivity of the small type 2 particles does not show this anomalous trend [see Fig. 10(b)]. From a qualitative perspective, these different behaviors perhaps might be expected since the larger particles begin to overlap more (and hence transition into anomalous mean-field behavior) at lower densities than the smaller particles. This aspect of binary Gaussian-core mixtures in discussed in detail elsewhere [31].

One consequence of the dynamic decoupling of small and large particles described above is a crossover density for tracer diffusivity. Specifically, large particles have lower tracer diffusivity than small particles at low density, but they attain higher values of tracer diffusivity than small particles at sufficiently high density [see Fig. 10(c)]. Because of this crossover, the Gaussian-core fluid mixture is an interesting counterexample to the fluids discussed thus far. It appears that both components cannot scale in a simple way with a single static measure like $-s^{\text {ex }}$ [Fig. 10(d)]. This is evident when one considers how the reduced Rosenfeld [Fig. 10(d)] and generalized Rosenfeld [Fig. 10(e)] forms of tracer diffusivity behave as a function of $-s^{\mathrm{ex}}$. As before, the original Rosenfeld form, $D_{i}^{\mathrm{R}}$, fails to collapse any of the data. The generalized Rosenfeld form, $D_{i}^{\mathrm{GR}}$, does an excellent job of collapsing the low-density data but necessarily breaks down 

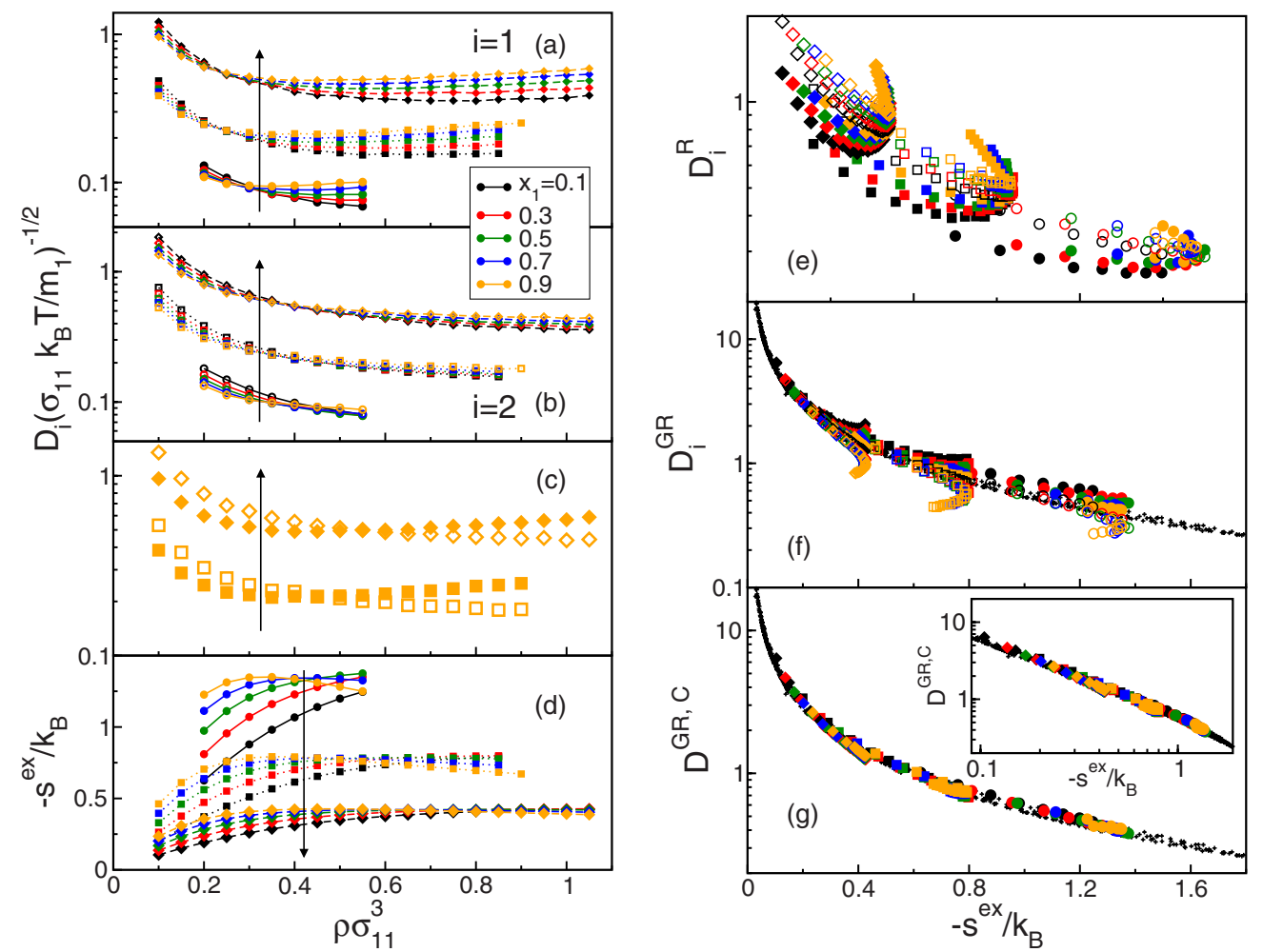

FIG. 10. (Color online) Properties of the binary Gaussian-core fluid described in the text. Tracer-diffusion coefficient of (a) component 1 and (b) component 2 versus density. (c) Illustration of the crossover in tracer diffusivity for components 1 and 2 as a function of density. (d) Excess-entropy $-s^{\text {ex }}$ versus density. (e) Rosenfeld scaled tracer-diffusivity $D_{i}^{\mathrm{R}}$, (f) generalized Rosenfeld tracer diffusivity $D_{i}^{\mathrm{GR}}$, and (g) collective generalized Rosenfeld tracer diffusivity $D^{\mathrm{GR}, \mathrm{C}}=\left(D_{i}^{\mathrm{GR}}\right)^{x_{1}}\left(D_{i}^{\mathrm{GR}}\right)^{x_{2}}$ versus $-s^{\mathrm{ex}}$. Symbol type corresponds to reduced temperature $k_{\mathrm{B}} T / \epsilon_{11}: 0.05$ (circles), 0.1 (squares), and 0.2 (diamonds). For clarity in (a)-(d), increasing temperature is given by the direction of the arrow. In (a)-(f), closed and open symbols denote components 1 and 2, respectively. Panel (c) displays the crossover behavior of the tracer diffusivities for $x_{1}=0.9$ and $k_{\mathrm{B}} T / \epsilon_{11}=0.05$ and 0.1 . In panels (f) and (g), small black crosses represent the single-component Gaussian-core data. Inset to $(\mathrm{g})$ is the same as the main plot but on a log-log scale.

at higher densities, where the anomalous behavior emerges.

We close our discussion of the binary Gaussian-core mixture with an interesting empirical observation. In the spirit of [60], we find that a collective tracer diffusivity of the mixture, which we define here as $D^{\mathrm{GR}, \mathrm{C}} \equiv\left(D_{i}^{\mathrm{GR}}\right)^{x_{1}}\left(D_{i}^{\mathrm{GR}}\right)^{x_{2}}$, is still a single valued of excess entropy over the wide range of temperature, density, and compositions investigated here [see Fig. $10(\mathrm{~g})]$. As can be seen, it also quantitatively tracks the relationship between $D^{\mathrm{R}}$ and $-s^{\mathrm{ex}}$ for the single-component Gaussian-core fluid. What this implies is that tracer diffusivity of one component can be predicted based on knowledge of tracer diffusivity of the other component, the excess entropy of the mixture, and the behavior of the one-component fluid. Of course, this observation also holds true (trivially) for the other mixtures we discussed earlier because the generalized Rosenfeld tracer diffusivities themselves are singlevalued functions of $s^{\mathrm{ex}}$ of those systems.

\section{CONCLUSIONS}

In this work, we present a dimensionless form of the tracer diffusion coefficient of a species which we call the generalized Rosenfeld tracer diffusivity. We show, via molecular simulation, that this quantity is approximately a single-valued function of excess entropy for a range of model one- and two-component fluid mixtures. The empirical excess entropy scaling is consistent with the various effects that composition, temperature, density, and microscopic interactions have on the equilibrium single-particle dynamics of these systems. Generalizing an earlier argument of [2], we show that the functional form of the reduced tracer diffusivity can be obtained by examining the theoretical behavior of excess entropy and tracer diffusivity in the low-particledensity limit.

We demonstrate that the aforementioned "generalized Rosenfeld" scaling applies more broadly than other simple approaches such as Enksog theory or empirical scalings based on the pair-correlation contribution to the excess entropy. However, we also identify some important limitations of the approach. For example, the scaling breaks down for highly asymmetric hard-sphere mixtures (diameter ratios of 5 or larger) for packing fractions near the freezing transition. It also breaks down for Gaussian-core mixtures, where the softness of the interactions combined with the size asymmetry gives rise to significant decoupling of the single-particle dynamics of the species. Interestingly, even in this latter case, we show that a single collective measure of the tracer diffusivities obeys an excess entropy scaling, which provides a 
quantitative link between structure and the tracer diffusivities of the two components.

It may also be fruitful in future work to develop generalized Rosenfeld scalings for other transport coefficients, such as thermal conductivity and shear viscosity. We plan to focus on extending the ideas of the present paper to systems with other types of dynamics (e.g., including effects of dissipation, hydrodynamic interactions, etc.). We also are studying what aspects of interparticle interactions can give rise to decoupling of species-specific structural and dynamic quantities.

\section{ACKNOWLEDGMENTS}

Two authors (T.M.T and J.R.E) acknowledge financial support of the National Science Foundation (Grants No. CTS-0448721 and No. CTS-028772, respectively). One author T.M.T. also acknowledges support of the Welch Foundation (F-1696) and the David and Lucile Packard Foundation. The Texas Advanced Computing Center (TACC) and the Biowulf Cluster at the National Institutes of Health provided computational resources for this paper.

\section{APPENDIX: EXTENSION TO BROWNIAN DYNAMICS}

Consider a collection of Brownian particles of radius $a$ and volume fraction $\varphi=4 \pi a^{3} \rho / 3$ suspended in a continuum solvent. We wish to identify a generalized Rosenfeld scaling of $D$, the long-time self-diffusion coefficient of the particles, which will be (approximately) a single-valued function of the excess entropy. In this case, since the solvent can be being treated as a continuum, the excess entropy of interest is that associated with the static correlations of the Brownian particles.

At infinite dilution, the excess entropy of the Brownian particles is zero, and $D$ is simply equal to the Stokes-Einstein diffusivity $D_{0}=k_{\mathrm{B}} T /(S \pi \eta a)$, where $S=4$ for slip and $S=6$ for stick boundary conditions, and $\eta$ is the solvent viscosity. To leading order in packing fraction $\varphi$, the difference between $D_{0}$ and $D$ can be expressed [61-64]

$$
\Delta D \equiv D_{0}-D=-D_{0} D_{2} \varphi,
$$

where $D_{2}$ characterizes how static correlations modify the long-time self-diffusivity of the particles. This quantity can be expressed as

$$
\begin{aligned}
D_{2}= & \int_{0}^{\infty}\left(-3+A_{11}+2 B_{11}\right) g(r) r^{2} d r \\
& +\int_{0}^{\infty}\left[\frac{A_{11}-A_{12}-B_{11}+B_{12}}{r}\right. \\
& \left.+\frac{1}{2}\left(\frac{d A_{11}}{d r}-\frac{d A_{12}}{d r}\right)\right] Q(r) g(r) r^{2} d r .
\end{aligned}
$$

Expressions for the functions $Q(r), A_{11}, A_{12}, B_{11}$, and $B_{12}$ are known in the hydrodynamic limit $[62,65]$. Also, in the dilute limit, one may further replace $g(r)$ in Eq. (A2) by the Boltzmann factor [Eq. (12)] of $V^{\prime}(r)$, the effective pair potential between the Brownian particles in solution.

Following Eq. (2), one can similarly express the excess entropy $s^{\prime \text { ex }}$ associated with the structure of the Brownian particles (to leading order in particle density) in terms of its osmotic second virial coefficient, $s^{\prime \mathrm{ex}}=-k_{\mathrm{B}} \rho\left[B^{\prime}\right.$ $\left.+d B^{\prime} / d \ln T\right]$. As in Eq. (3b), $B^{\prime}$ can be readily obtained from knowledge of $V^{\prime}(r)$. Using the osmotic virial expression for the excess entropy to eliminate packing fraction from Eq. (A1) and rearranging yields a Brownianappropriate generalized Rosenfeld self-diffusivity:

$$
\Delta D^{\mathrm{GR}} \equiv \frac{\left(D_{0}-D\right)\left[B^{\prime}+T \frac{d B^{\prime}}{d T}\right]}{-D_{0} D_{2} \frac{4}{3} \pi a^{3}}=-s^{\prime \mathrm{ex}} / k_{\mathrm{B}} .
$$

Although the quantity, $\Delta D^{\mathrm{GR}}$ is strictly a single-valued function of $s^{\prime \text { ex }}$ in the dilute limit, its behavior at higher particle concentrations needs to be studied further.
[1] Y. Rosenfeld, Phys. Rev. A 15, 2545 (1977).

[2] Y. Rosenfeld, J. Phys.: Condens. Matter 11, 5415 (1999).

[3] M. Dzugutov, Nature (London) 381, 137 (1996).

[4] J. Mittal, J. Errington, and T. Truskett, J. Phys. Chem. B 111, 10054 (2007).

[5] E. H. Abramson, Phys. Rev. E 76, 051203 (2007).

[6] E. H. Abramson and H. West-Foyle, Phys. Rev. E 77, 041202 (2008).

[7] E. H. Abramson, Phys. Rev. E 80, 021201 (2009).

[8] J. Mittal, J. R. Errington, and T. M. Truskett, Phys. Rev. Lett. 96, 177804 (2006).

[9] J. Mittal, J. R. Errington, and T. M. Truskett, J. Chem. Phys. 126, 244708 (2007).

[10] J. Mittal, V. K. Shen, J. R. Errington, and T. M. Truskett, J. Chem. Phys. 127, 154513 (2007).

[11] G. Goel, W. P. Krekelberg, M. J. Pond, J. Mittal, V. K. Shen, J.
R. Errington, and T. M. Truskett, J. Stat. Mech.: Theory Exp. 2009, P04006 (2009).

[12] G. Goel, W. P. Krekelberg, J. R. Errington, and T. M. Truskett, Phys. Rev. Lett. 100, 106001 (2008).

[13] J. R. Errington and P. G. Debenedetti, Nature (London) 409, 318 (2001).

[14] M. S. Shell, P. G. Debenedetti, and A. Z. Panagiotopoulos, Phys. Rev. E 66, 011202 (2002).

[15] T. M. Truskett and K. A. Dill, J. Phys. Chem. B 106, 11829 (2002).

[16] P. Kumar, S. V. Buldyrev, F. Sciortino, E. Zaccarelli, and H. E. Stanley, Phys. Rev. E 72, 021501 (2005).

[17] R. Esposito, F. Saija, A. M. Saitta, and P. V. Giaquinta, Phys. Rev. E 73, 040502(R) (2006).

[18] P. A. Netz, S. V. Buldyrev, M. C. Barbosa, and H. E. Stanley, Phys. Rev. E 73, 061504 (2006). 
[19] L. Xu, S. V. Buldyrev, C. A. Angell, and H. E. Stanley, Phys. Rev. E 74, 031108 (2006).

[20] J. Mittal, J. R. Errington, and T. M. Truskett, J. Phys. Chem. B 110, 18147 (2006).

[21] J. R. Errington, T. M. Truskett, and J. Mittal, J. Chem. Phys. 125, 244502 (2006).

[22] R. Sharma, S. N. Chakraborty, and C. Chakravarty, J. Chem. Phys. 125, 204501 (2006).

[23] A. B. de Oliveira, M. C. Barbosa, and P. A. Netz, Physica A 386, 744 (2007).

[24] Z. Yan, S. V. Buldyrev, P. Kumar, N. Giovambattista, P. G. Debenedetti, and H. E. Stanley, Phys. Rev. E 76, 051201 (2007).

[25] M. M. Szortyka and M. C. Barbosa, Physica A 380, 27 (2007).

[26] A. B. de Oliveira, G. Franzese, P. A. Netz, and M. C. Barbosa, J. Chem. Phys. 128, 064901 (2008).

[27] W. P. Krekelberg, J. Mittal, V. Ganesan, and T. M. Truskett, Phys. Rev. E 77, 041201 (2008).

[28] Z. Yan, S. V. Buldyrev, and H. E. Stanley, Phys. Rev. E 78, 051201 (2008).

[29] W. P. Krekelberg, J. Mittal, V. Ganesan, and T. M. Truskett, J. Chem. Phys. 127, 044502 (2007).

[30] W. P. Krekelberg, T. Kumar, J. Mittal, J. R. Errington, and T. M. Truskett, Phys. Rev. E 79, 031203 (2009).

[31] M. J. Pond, W. P. Krekelberg, V. K. Shen, J. R. Errington, and T. M. Truskett, J. Chem. Phys. 131, 161101 (2009).

[32] A. Chaimovich and M. S. Shell, Phys. Chem. Chem. Phys. 11, 1901 (2009).

[33] W. G. Hoover, Computational Statistical Mechanics (Elsevier, New York, 1991), pp. 172-173.

[34] N. Gnan, T. B. Schrder, U. R. Pedersen, N. P. Bailey, and J. C. Dyre, e-print arXiv:0905.3497.

[35] A. Lang, C. N. Likos, M. Watzlawek, and H. Lowen, J. Phys.: Condens. Matter 12, 5087 (2000).

[36] A. A. Louis, P. G. Bolhuis, and J. P. Hansen, Phys. Rev. E 62, 7961 (2000).

[37] C. N. Likos, Phys. Rep. 348, 267 (2001).

[38] P. Mausbach and H.-O. May, Z. Phys. Chem. 223, 1035 (2009).

[39] A. Samanta, Sk. Musharaf Ali, and S. K. Ghosh, Phys. Rev. Lett. 87, 245901 (2001).

[40] The focus of the present paper is on molecular dynamics, where the particles obey Newton's equations of motion. In the Appendix, we illustrate how this analysis might be extended to treat the dissipative dynamics relevant for Brownian particles suspended in a solvent.
[41] S. Chapman and T. G. Cowling, The Mathematical Theory of Nonuniform Gases, 3rd ed. (Cambridge University Press, 1970).

[42] G. Jacucci and I. R. McDonald, Physica A 80, 607 (1975).

[43] K. Tankeshwar and F. Ould-Kaddour, J. Phys.: Condens. Matter 4, 3349 (1992).

[44] S. K. Sharma and K. Tankeshwar, J. Phys.: Condens. Matter 8, 10839 (1996).

[45] K. Tankeshwar, B. Singla, and K. N. Pathak, J. Phys.: Condens. Matter 3, 3173 (1991).

[46] B. Widom and J. S. Rowlinson, J. Chem. Phys. 52, 1670 (1970).

[47] F. H. Stillinger and T. A. Weber, J. Chem. Phys. 68, 3837 (1978).

[48] A. J. Archer and R. Evans, Phys. Rev. E 64, 041501 (2001).

[49] D. C. Rapaport, The Art of Molecular Dynamic Simulation, 2nd ed. (Cambridge University Press, Cambridge, 2004).

[50] M. P. Allen and D. J. Tildesley, Computer Simulations of Liquids (Oxford University Press, New York, 1987).

[51] V. K. Shen and J. R. Errington, J. Chem. Phys. 124, 024721 (2006).

[52] V. K. Shen and J. R. Errington, J. Chem. Phys. 122, 064508 (2005).

[53] T. Boublik, J. Chem. Phys. 53, 471 (1970).

[54] G. A. Mansoori, N. F. Carnahan, K. E. Starling, and T. W. Leland, J. Chem. Phys. 54, 1523 (1971).

[55] I. Biazzo, F. Caltagirone, G. Parisi, and F. Zamponi, Phys. Rev. Lett. 102, 195701 (2009).

[56] S. Yerazunis, S. W. Cornell, and B. Wintner, Nature (London) 207, 835 (1965).

[57] J. A. Hernando, Mol. Phys. 69, 319 (1990).

[58] P. Mausbach and H. O. May, Fluid Phase Equilib. 249, 17 (2006).

[59] H. Wensink, H. Löwen, M. Rex, C. Likos, and S. van Teeffelen, Comput. Phys. Commun. 179, 77 (2008).

[60] J. J. Hoyt, M. Asta, and B. Sadigh, Phys. Rev. Lett. 85, 594 (2000).

[61] B. Cichocki and B. U. Felderhof, J. Chem. Phys. 93, 4427 (1990).

[62] B. Cichocki and B. U. Felderhof, J. Chem. Phys. 89, 3705 (1988).

[63] G. K. Batchelor, J. Fluid Mech. 131, 155 (1983).

[64] G. K. Batchelor, J. Fluid Mech. 74, 1 (1976).

[65] D. J. Jeffrey and Y. Onishi, J. Fluid Mech. 139, 261 (1984). 